\title{
Mitochondrial Diabetes Treated With a Sodium-Glucose Co-Transporter 2 Inhibitor
}

\author{
Hidetaka Hamasaki a, b, c
}

\begin{abstract}
Recent animal studies have suggested that sodium-glucose co-transporter 2 (SGLT2) inhibitors have mitochondrial and cardiorenal protective effects. Herein we report the case of mitochondrial diabetes successfully treated with an SGLT2 inhibitor empagliflozin. Although the underlying mechanisms have still not been completely investigated, SGLT2 inhibitors may improve mitochondrial function, suppress oxidative stress and restore autophagy. SGLT2 inhibitors could be a treatment option for patients with mitochondrial diabetes.
\end{abstract}

Keywords: Mitochondrial diabetes; SGLT2 inhibitor; Empagliflozin

\section{Introduction}

Mitochondrial diabetes, also known as maternal-inherited diabetes with deafness, is characterized by a gradual progression of pancreatic $\beta$-cell dysfunction upon aging. The development of mitochondrial diabetes is associated with m.3243A $>\mathrm{G}$ mutation that affects insulin secretion via decreased ATP synthesis [1]. Although some agents such as coenzyme Q10 [2] potentially have a therapeutic effect on mitochondrial disease, the early use of insulin injection is commonly required to treat mitochondrial diabetes due to insulin deficiency.

Sodium-glucose co-transporter 2 (SGLT2) inhibitors are oral glucose-lowering agents that have beneficial effects on cardiovascular disease [3-5]. Recently, several studies have shown the pleiotropic effects of SGLT2 inhibitors. Notably, SGLT2 inhibitors may exert mitochondrial and cardiorenal protective effects by inhibiting complex I activity in the mitochondrial electron transport system [6]. SGLT2 inhibitors might be useful in treating mitochondrial diabetes.

Herein we report the case of mitochondrial diabetes successfully treated with an SGLT2 inhibitor empagliflozin and discuss the mechanism of its protective effect on mitochondrial function.

Manuscript submitted December 5, 2019, accepted December 13, 2019

${ }^{a}$ Hamasaki Clinic, Kagoshima, Japan

bDepartment of Diabetes, Imakiire General Hospital, Kagoshima, Japan

${ }^{\mathrm{c} C o r r e s p o n d i n g ~ A u t h o r: ~ H i d e t a k a ~ H a m a s a k i, ~ H a m a s a k i ~ C l i n i c, ~ 2-21-4 ~ N i s h i-~}$

da, Kagoshima 890-0046, Japan. Email: h-hamasaki@umin.ac.jp

doi: https://doi.org/10.14740/jem627

\section{Case Report}

A 36-year-old man visited our hospital in April 2019 after being diagnosed with hyperglycemia during his annual medical checkup. At the age of 52 years, his mother died of mitochondrial encephalomyopathy with lactic acidosis and stroke-like episodes; therefore, he requested for a detailed examination. His height and weight were $172.5 \mathrm{~cm}$ and $74.5 \mathrm{~kg}$, respectively. His fasting plasma glucose and hemoglobin A1c levels had increased to $190 \mathrm{mg} / \mathrm{dL}$ and $11.0 \%$, respectively, at the first visit. He showed negative results for anti-glutamic acid decarboxylase antibody, anti-insulinoma-associated antigen-2 antibody and anti-insulin antibody. However, his urinary C-peptide levels had decreased to 44.9 (normal range: 59 - 140) $\mu \mathrm{g} /$ day. In addition, his blood lactate-to-pyruvate ratio increased to 16.5 (lactate and pyruvate levels were 19.1 and $1.16 \mathrm{mg} / \mathrm{dL}$, respectively). Although he did not present with neuromuscular manifestations and deafness, mitochondrial DNA sequence analysis revealed m.3243A $>$ G mutation. He was diagnosed with mitochondrial diabetes; therefore, insulin therapy combined with diet therapy was initiated. As a result of taking three units of insulin aspart before breakfast and dinner and four units of insulin glargine before bedtime, his blood glucose level became stable. Furthermore, HbA1c levels decreased to $7.1 \%$ in August. A $10 \mathrm{mg} /$ day dosage of empagliflozin, an SGLT2 inhibitor, was added to the insulin therapy, and the treatment using insulin aspart was discontinued. HbA1c levels further decreased to $6.6 \%$ in September, and blood lactate-to-pyruvate ratio decreased to 14.4 (lactate and pyruvate levels were 12.1 and $0.84 \mathrm{mg}$ / $\mathrm{dL}$, respectively). Finally, insulin therapy was completed. Two months after the administration of empagliflozin, he maintained good glycemic control (HbA1c level, 6.5\%) without any adverse effects, and his body weight did not reduce $(76.9 \mathrm{~kg})$.

\section{Discussion}

To the best of my knowledge, this is the first report to demonstrate the efficacy and safety of empagliflozin monotherapy for treating a patient with mitochondrial diabetes. The blood lactate-to-pyruvate ratio might have decreased due to the administration of empagliflozin. SGLT2 inhibitors might be a promising drug class for treating mitochondrial diabetes.

Mizuno et al [7] recently showed that treatment with empagliflozin normalizes the size and number of mitochondria as well as prevents the reduction in the number of mitochondria 
after myocardial infarction in cardiomyocytes of diabetic rats. The study suggested that the administration of empagliflozin reduced the level of reactive oxygen species and restored autophagy. In addition, Takagi et al [8] demonstrated that ipragliflozin restores morphological mitochondrial damage in renal tubules and improves mitochondrial function in mice with high-fat diet. Furthermore, a study by Durak et al [9] suggested that dapagliflozin exhibited a cardioprotective effect via the augmentation of mitochondrial function and suppression of oxidative stress in rats with high-carbohydrate diet-induced metabolic syndrome. Although the underlying mechanism has still not been completely investigated, SGLT2 inhibitors may improve mitochondrial function.

Mitochondrial dysfunction can lead to insulin resistance and secretion impairment. Patients with $\mathrm{m} .3243 \mathrm{~A}>\mathrm{G}$ and diabetes usually have insulin resistance as well as insulin deficiency. Previous studies reported that only $13 \%$ patients with $\mathrm{m} .3243 \mathrm{~A}>\mathrm{G}$ mutation and diabetes required insulin therapy at disease onset [10]. In this case, insulin secretion did not completely decrease; therefore, the patient could achieve glycemic control with low-dose insulin therapy. The pathophysiological condition of the patient was supposed to be attributed to both insulin resistance and insulin deficiency. The administration of SGLT2 inhibitors might improve insulin resistance through the improvement of mitochondrial function in addition to fat utilization, browning of white adipose tissue and reducing obesityinduced inflammation [11]. However, to elucidate the underlying mechanism, further research is required.

\section{Acknowledgments}

The author appreciates the support of Rika Fukumura, Kayoko Takakura and Kayoko Kajiya for their help in patient care.

\section{Financial Disclosure}

None to declare.

\section{Conflict of Interest}

The author declares no conflict of interest.

\section{Informed Consent}

Written informed consent was obtained from the patient for publication of this case report.

\section{Author Contributions}

Study design: Hidetaka Hamasaki. Data collection: Hidetaka
Hamasaki. Data interpretation: Hidetaka Hamasaki. Manuscript preparation: Hidetaka Hamasaki. Literature search: Hidetaka Hamasaki.

\section{Data Availability}

The author declares that data supporting the findings of this study are available within the article.

\section{References}

1. Maassen JA, LM TH, Van Essen E, Heine RJ, Nijpels G, Jahangir Tafrechi RS, Raap AK, et al. Mitochondrial diabetes: molecular mechanisms and clinical presentation. Diabetes. 2004;53(Suppl 1):S103-109.

2. Hernandez-Camacho JD, Bernier M, Lopez-Lluch G, Navas P. Coenzyme Q10 supplementation in aging and disease. Front Physiol. 2018;9:44.

3. Zinman B, Wanner C, Lachin JM, Fitchett D, Bluhmki E, Hantel S, Mattheus M, et al. Empagliflozin, cardiovascular outcomes, and mortality in type 2 diabetes. N Engl J Med. 2015;373(22):2117-2128.

4. Neal B, Perkovic V, Mahaffey KW, de Zeeuw D, Fulcher G, Erondu N, Shaw W, et al. Canagliflozin and cardiovascular and renal events in type 2 diabetes. N Engl J Med. 2017;377(7):644-657.

5. Wiviott SD, Raz I, Bonaca MP, Mosenzon O, Kato ET, Cahn A, Silverman MG, et al. Dapagliflozin and cardiovascular outcomes in type 2 diabetes. N Engl J Med. 2019;380(4):347-357.

6. Lee HK. Cardiorenal protective effect of sodium-glucose cotransporter 2 inhibitors and mitochondrial function. J Diabetes Investig. 2019;10(3):557-559.

7. Mizuno M, Kuno A, Yano T, Miki T, Oshima H, Sato T, Nakata $\mathrm{K}$, et al. Empagliflozin normalizes the size and number of mitochondria and prevents reduction in mitochondrial size after myocardial infarction in diabetic hearts. Physiol Rep. 2018;6(12):e13741.

8. Takagi S, Li J, Takagaki Y, Kitada M, Nitta K, Takasu T, Kanasaki K, et al. Ipragliflozin improves mitochondrial abnormalities in renal tubules induced by a high-fat diet. J Diabetes Investig. 2018;9(5):1025-1032.

9. Durak A, Olgar Y, Degirmenci S, Akkus E, Tuncay E, Turan B. A SGLT2 inhibitor dapagliflozin suppresses prolonged ventricular-repolarization through augmentation of mitochondrial function in insulin-resistant metabolic syndrome rats. Cardiovasc Diabetol. 2018;17(1):144.

10. Chow J, Rahman J, Achermann JC, Dattani MT, Rahman S. Mitochondrial disease and endocrine dysfunction. Nat Rev Endocrinol. 2017;13(2):92-104.

11. $\mathrm{Xu} \mathrm{L}, \mathrm{Ota} \mathrm{T}$. Emerging roles of SGLT2 inhibitors in obesity and insulin resistance: Focus on fat browning and macrophage polarization. Adipocyte. 2018;7(2):121128. 